\title{
Viscoelasticity Modeling of the Prostate Region Using Vibro-elastography
}

\author{
S.E. Salcudean ${ }^{1}$, Daniel French ${ }^{1}$, S. Bachmann ${ }^{1}$, R. Zahiri-Azar ${ }^{1}$, \\ X. Wen ${ }^{1}$, and W.J. Morris ${ }^{2}$ \\ ${ }^{1}$ Department of Electrical and Computer Engineering, University of British \\ Columbia, Canada \\ tims@ece.ubc.ca \\ ${ }^{2}$ Radiation Oncology, British Columbia Cancer Agency, Canada
}

\begin{abstract}
We present an ultrasound vibro-elastography system designed to acquire viscoelastic properties of the prostate and peri-prostatic tissue. An excitation stage imparts low-frequency $(<20 \mathrm{~Hz})$, limited amplitude $(< \pm 2 \mathrm{~mm})$, broadband vibratory motion to an endorectal transducer, along a radial/transversal direction. The induced tissue motion is estimated from ultrasound radio-frequency data and is used to estimate the mechanical frequency response of tissue to the excitation at different spatial locations. This can be used to determine the spatial distribution of various mechanical parameters of tissue, such as stiffness and viscosity. Phantom and in-vivo images are presented. The results obtained demonstrate high phantom and tissue linearity and high signal-to-noise ratio.
\end{abstract}

\section{Introduction}

Brachytherapy is a widely used treatment for prostate cancer. The treatment consists of the placement of radioactive seeds in the prostate using trans-perineal insertion of needles, in a pattern that delivers a sufficient radiation dose to kill the cancer while maintaining a tolerable dose to the urethra and rectum [1]. Biochemical and clinical relapse rates are very low with current brachytherapy procedures. However, improvements are still required to reduce the rate of complications, which include erectile dysfunction, urinary retention, incontinence and rectal injuries. Complications have been associated with high dose loads to large portions of the prostate, with high urethral dose and with needle trauma. Complication rates may be reduced by more accurate needle insertions.

Seed placement errors are caused by poor visualization of the prostate and by prostate movement due to needle forces. B-mode ultrasound (US) and X-ray fluoroscopy are typically used for image guidance during brachytherapy. Prostate segmentation in US images is unreliable, whether done manually or automatically 2, especially at the apex where the prostate blends into the muscles of the pelvic floor, and at the base, where the anterior portion is iso-echoic with the neck of the bladder. During each needle insertion, needle forces move the prostate 3] and cause seed placement errors 4. Prostate swelling and deformation caused by edema, and motion due to respiration also generate errors $[5]$. 
Thus there is a need to reliably localize the prostate based on US images and to predict tissue deformation due to needle forces.

The use of tissue elastic models for needle insertion planning has been proposed before. In 6], rigid needle insertion optimization based on a 2-D linear Finite-Element Method (FEM) tissue model has been proposed. In 7], needle motion planning based on 2-D FEM tissue and flexible needle models is reported. Needle targeting using discrete spring models is described in [8]. All these methods require knowledge of the tissue elastic properties, which are also required for the development of medical simulators 9 .

The elastic properties of the prostate have been measured before ex-vivo [10]. These can also be extracted from strain imaging systems, such as presented in 11 for prostate cancer detection and image-guided biopsy [12. However, traditional strain imaging systems with quasi-static and/or manual excitation produce images that have significant variance and are operator dependent.

This paper presents a system for the acquisition of patient-specific elastic properties of tissue in the prostate region using a dynamic ultrasound elastography method called vibro-elastography (VE) [13]. The system produces stable, operator-independent images that can be used to estimate stiffness as well as other properties of tissue. The hardware, signal processing and software necessary for this purpose are presented in Section 2 of this paper. Phantom and in-vivo imaging results are presented in Section 3, followed by a discussion of the results in Section 4 and conclusions in Section 5.

\section{Methods}

In VE, tissue is compressed by a vibrating actuator driven by low-pass filtered white noise, typically up to $20 \mathrm{~Hz}$, while tissue is imaged with US over a short time interval (1-10 s) (Fig. 1). The US radio-frequency (RF) data is used to determine time sequences of tissue displacements $x_{i}(t)$ at spatial locations $i$, typically along the same US RF A-line. Assuming that the response of tissue to the excitation is linear, mechanical frequency responses or transfer functions (TFs) $H_{i}^{j}(\omega), \omega \in \mathbb{R}$ of the displacement at spatial location $j$ with respect to the displacement at spatial location $i$ viewed as an input, and the associated coherence functions $C_{i}^{j}(\omega)$, can be computed by well-known techniques [14]:

$$
H_{i}^{j}(\omega)=P_{x_{i} x_{j}}(\omega) / P_{x_{i} x_{i}}(\omega) ; \quad C_{i}^{j}(\omega)=\left|P_{x_{i} x_{j}}(\omega)\right|^{2} /\left(P_{x_{i} x_{i}}(\omega) P_{x_{j} x_{j}}(\omega)\right) .
$$

where $P_{x_{i} x_{j}}(\omega)$ denotes the cross spectral density between $x_{i}(t)$ and $x_{j}(t) . C_{i}^{j}(\omega) \in$ $[0,1]$ indicates the proportion of input energy at location $i$ at $\omega$ that appears at the output $j$ at the same $\omega$ and therefore it is an indicator of both system linearity and signal-to-noise ratio. One has high confidence in the TF estimate $H_{i}^{j}(\omega)$ if the coherence function $C_{i}^{j}(\omega)$ is close to 1 at $\omega$.

Mechanical tissue properties such as stiffness and viscosity can be derived from these TFs. For example, $\left|H_{i}^{0}(0)\right|$ estimates the static deformation at location $i$ due to a unit displacement at location 0 . Therefore $\left|H_{i}^{i+1}(\omega) / H_{i-1}^{i}(\omega)\right|_{\omega=0}$ will 


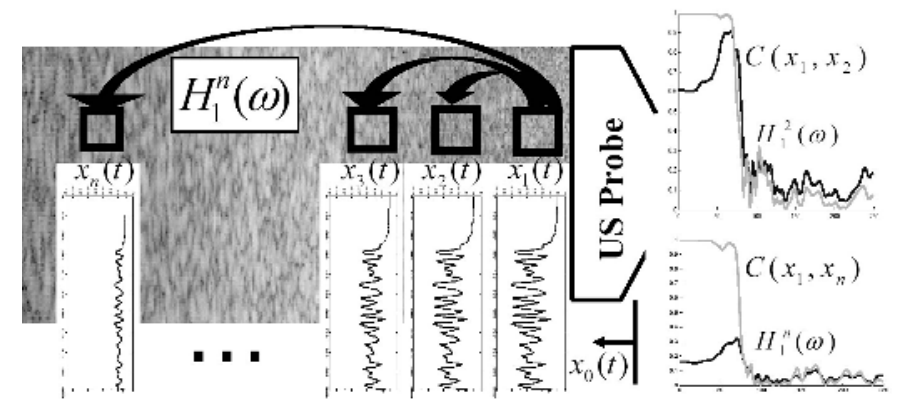

Fig. 1. Vibro-elastography with actuated US transducer, measured displacements, frequency responses and coherence functions

show relative tissue compliance between consecutive spatial locations. Stiffness estimation based on TFs relies on elastic behavior that can be averaged over an entire low frequency range and therefore it is superior to static elastograms [13. Stiffness changes of $10 \%$ and stiff $(2 \mathrm{X})$ enclosures of $0.7 \mathrm{~mm}$ can be detected with a linear $8 \mathrm{MHz}$ transducer [13. A trans-rectal US (TRUS) VE system has been constructed. The system utilizes an Ultrasonix 500 RP US machine with a biplane TRUS transducer (Ultrasonix Medical Corp.). The transducer is mounted on an actuating system, described in Section 2.1, which is used to apply compression waves to the rectal wall. US images are acquired and processed on the Ultrasonix 500 RP PC-based machine using the signal processing techniques described below.

\subsection{Vibro-elastography Actuation Stage Design}

The TRUS VE actuation system can translate and orient the TRUS transducer and can impart to it radial vibratory motion (Fig. 2). It has three main parts: a vibrating mechanism, a rotational stage and a transverse stage. The maximum displacement of the endorectal probe from its nominal center can be set by limit stops in the range $\pm 0-5 \mathrm{~mm}$, while experimental results have shown that $\pm 2 \mathrm{~mm}$ is sufficient for the acquisition of prostate images. The actuation system is mounted on a standard brachytherapy stabilizer for coarse positioning.

Low inertia coreless motors (\#118778 Maxon Precision Motors) with digital encoders (QPhase 3076A007-5000-0, Quantum Devices Inc), current amplifiers and a PCI interface card (LCAM and Q8, Quanser Inc.) have been used in the actuation system. A PC-based real-time system (WinCon by Quanser and Simulink from The MathWorks) and a user interface that allows desired waveforms to be executed have been implemented. The closed loop position control bandwidth achieved with the present controller is of $10 \mathrm{~Hz}$ for small amplitude motion, while a pre-compensator allows open loop position excitation at much higher frequencies. A number of safety features have been implemented and include system kill switches for two system operators, fused motors, software limit stops and procedures that enforce a properly initialized start-up sequence. 

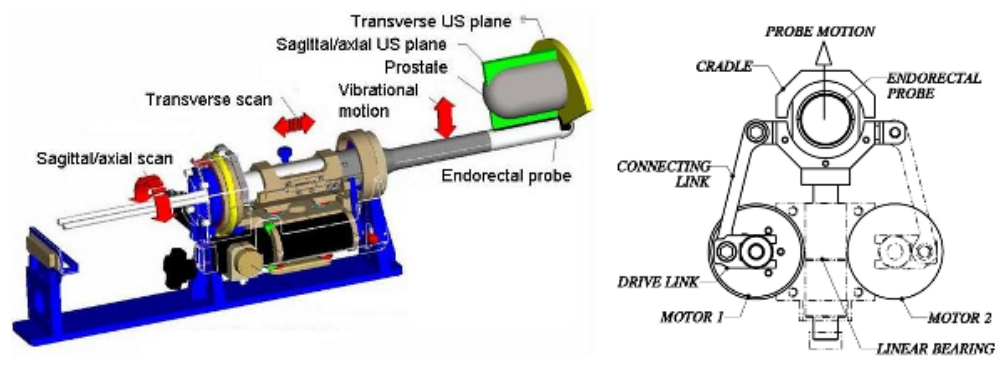

Fig. 2. VE actuation system schematic (left) and transversal schematic view of the vibratory system (right). The dual imaging probe (imaging planes shown through the prostate model) is mounted in a housing and is vibrated along its axial plane by two motor-driven four-bar linkages, along a direction set by the rotational actuator, with its insertion depth adjusted by the translational actuator. Volume images can be acquired using the transverse or the sagittal imaging planes.

\subsection{Vibro-elastography Signal Processing}

Real-time Motion Estimation. Tissue motion due to TRUS probe compression and relaxation is estimated by time domain cross-correlation. Each A-line of RF data is divided into $N$ short, equal-length overlapping windows. The displacement $x_{i}(k T)$ of the $i^{\text {th }}$ window from sample time $k T$ to sample time $k T+T$ can be obtained by maximizing its cross-correlation to the RF A-line sampled at time $k T+T$. Because adjacent segments have similar displacements when the US image sampling period $T$ is small, the search for cross-correlation maximization can be bracketed to a very small predicted interval 15. Sub-sample resolution is achieved by interpolation (quadratic, cosine). The normalized crosscorrelation is monitored. When it drops below a certain threshold (e.g., 0.9), a "recovery search" that samples a larger segment of the RF A-line, not just the small predicted interval, is performed. While we have not carried out an extensive evaluation during brachytherapy, in phantom studies and limited in-vivo studies, the recovery search is seldom triggered - in fewer than $2 \%$ of processed windows, allowing processing of windows of 16,000 pixels at $30 \mathrm{~Hz}$.

Real-time Frequency Response Estimation. The displacements $\left\{x_{i}(k T)\right\}$ of the $i^{\text {th }}$ A-line RF data window are buffered in segments of fixed length and overlap. Estimates of $H_{M}^{i}(\omega), i=1, \ldots, M-1, M+1, \ldots, N$ from a reference A-line RF window to each of the other windows in the A-line are obtained using Welch's periodogram method [16], in which the spectral densities are efficiently updated every time a new data segment is accumulated. The periodic updates are obtained by using all the data, by discarding old data or by discounting the effect of old data exponentially using a "forgetting factor". Discarding or discounting old data results in TF update based on recent data which enables the operator to make real-time adjustments to the VE imaging system.

Display of Images. VE generates a spatial distribution of frequency responses with a wide choice of parameters for display. In all images presented in this paper we display the $L_{2}$ norm of the differences between TFs at different spatial 
locations $\left\|W\left(H_{i}^{i+1}-H_{i-1}^{i}\right)\right\|_{2}=\left\|W\left(H_{M}^{i+1} / H_{M}^{i}-H_{M}^{i} / H_{M}^{i-1}\right)\right\|_{2}$, where $W$ is a frequency-dependent weight matching the input excitation frequency. In the low range of excitation frequencies considered in this paper, the acquired responses are fairly flat and therefore this norm approximates tissue compliance.

\subsection{Phantom Construction}

To tune and characterize the TRUS VE system, a prostate phantom has been constructed. It consists of a rubber balloon filled with $14 \%$ gelatin gel and $2 \%$ cellulose (by mass) (G9382-B gelatin, S-5504 cellulose, Sigma-Aldrich Inc.). The balloon is embedded in $10 \mathrm{~cm} \times 10 \mathrm{~cm} \times 10 \mathrm{~cm}$ substrate of $10 \%$ gelatin gel and $2 \%$ cellulose. The gel in the balloon is approximately twice as stiff as the substrate and has similar echogenic properties. A cylindrical hole in the phantom mimics the rectum and permits insertion of the endorectal ultrasound probe.

\section{Results}

In the images presented, the RF A-lines are split into 80 windows each with $50 \%$ window overlap. Axial images contain $128 \mathrm{RF}$ A-lines along the $60 \mathrm{~mm}$ long transducer crystal. Transverse images contain $114 \mathrm{RF}$ A-lines. Image depth is
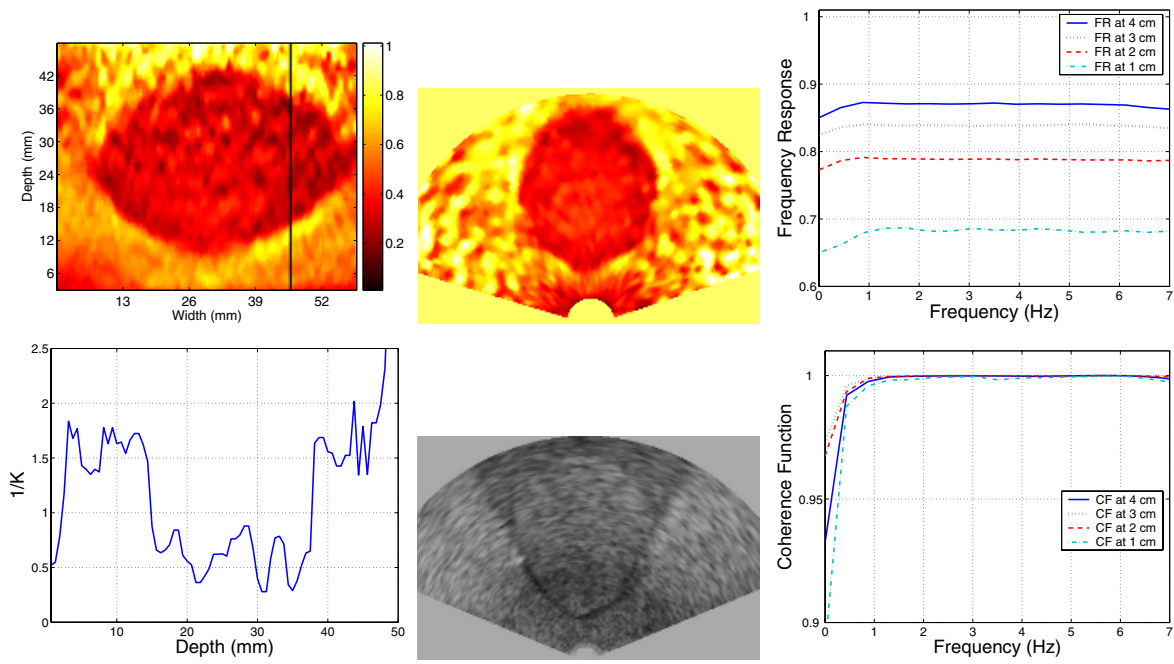

Fig. 3. Prostate phantom images. Sagittal VE image (top left), relative compliance along the marked VE A-line showing a change of a factor of 2 (bottom left - inclusion is stiffer), transversal images (top middle - VE, bottom middle - B-mode), TFs (top right) and corresponding coference functions (bottom right) along the marked A-line are displayed. The motion applied is $4 \mathrm{~mm}$ peak-to-peak, band-pass filtered $(0.5-10 \mathrm{~Hz})$ white noise. Transfer functions are averaged over 10 seconds, and are computed with reference to the motion in the focal area of the firing crystal at a depth of $25 \mathrm{~mm}$. Colour coding is HOT as defined in Matlab - see index bar. 

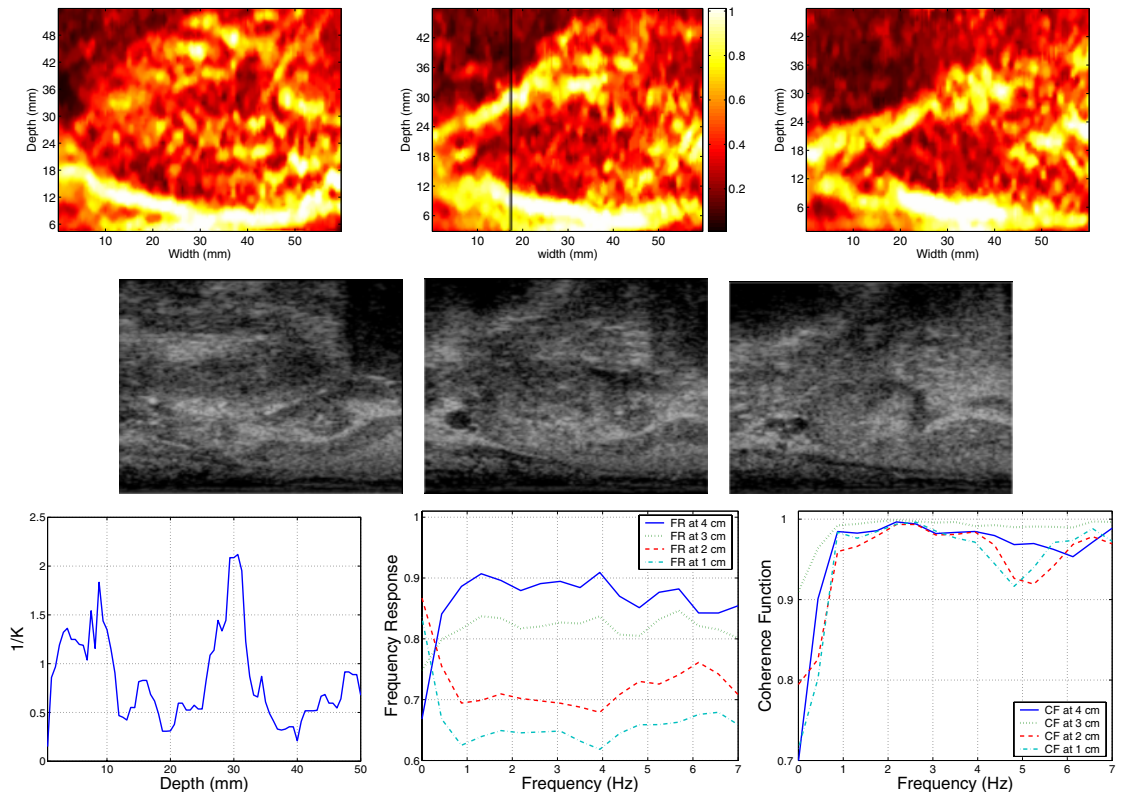

Fig. 4. Prostate sagittal and para-sagittal images near the apex. VE - top row, showing sagittal plane (at 90 degrees), and para-sagittal planes at 80 degrees and 70 degrees. The corresponding B-mode images are shown in the middle row. The bottom row shows the relative compliance (left), five TFs (middle) and the corresponding coherence functions (right) along the marked A-line. The motion applied was $2 \mathrm{~mm}$ peak-to-peak, bandpass filtered (0.5-4.5 Hz) white noise. TFs are computed with reference to a window at $25 \mathrm{~mm}$ depth.

$50 \mathrm{~mm}$ in all cases, transducer frequency is $5 \mathrm{MHz}$, and sampling frequency is $40 \mathrm{MHz}$. The RF-data acquisition and processing frame rate was $14 \mathrm{~Hz}$. TFs were displayed at $2 \mathrm{~Hz}$, allowing real-time tuning of imaging parameters (e.g., compression, amplitude, and frequency content of the input).

Images of the phantom described above are shown in Fig. 3, A protocol for acquiring VE images from patients has been designed and images have been acquired from three patients in the operating room just prior to the brachytherapy procedure. Images are presented in Fig. 4 and Fig. 5. 1D median filtering with a kernel of 5 samples has been applied to displacement estimates, which show a normalized correlation coefficient above 0.9 in over $90 \%$ of windows. A 2D median filter with a $3 \times 3$ pixel kernel has been applied to VE images, which are also decay-compensated in a standard manner.

\section{Discussion and Future Work}

The VE images obtained, as shown in Figs. 3, 4, and 5, show very good delineation of the prostate gland by comparison to B-mode US images. Furthermore, they show significant and repeatable details, including stiff regions within the 

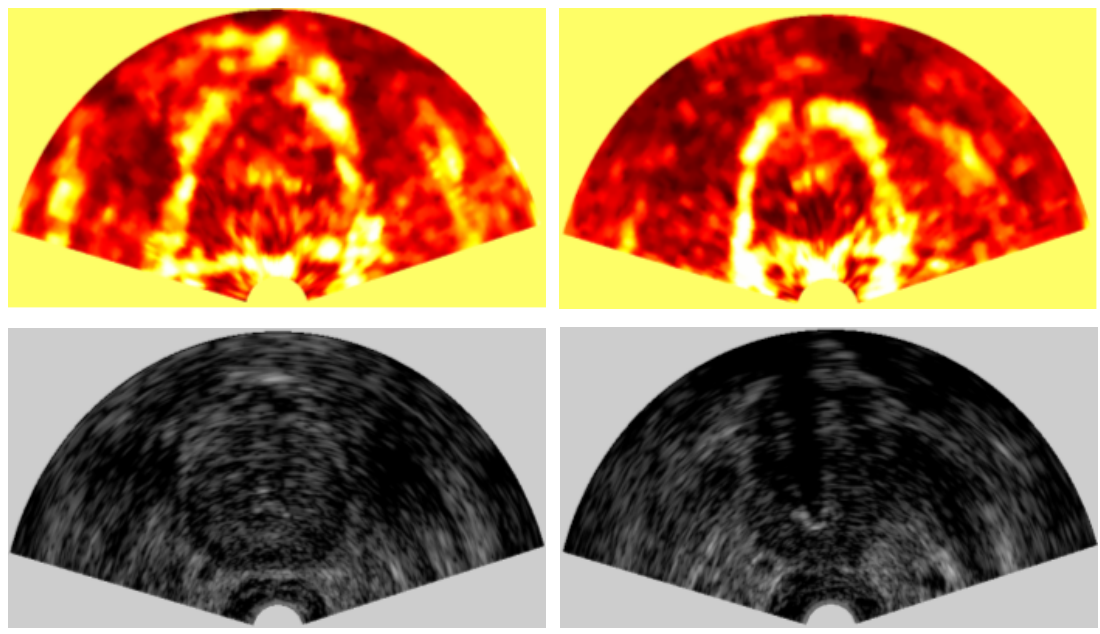

Fig. 5. Prostate transversal images. Top row shows VE images of the midgland (left) and apex (right), bottom row corresponding B-mode images. The motion applied was the same as in Fig. 4 TFs were computed with reference to a window at $25 \mathrm{~mm}$ depth.

prostate. The results show surprisingly high coherence over the frequencies of excitation, over 0.9 in $80 \%$ of TFs shown in the images. While the excitation frequency is quite low, this indicates good confidence in the measurement.

For good estimation of the transfer function, there must be sufficient frequency content in the transducer motion which provides the tissue compression. In this work, the motion of a window (indexed by $M$ ) in the focal area of the A-line was used as a reference. While this motion may not be as "white" as the transducer motion which is under computer control, we have not synchronized yet the US transducer location to the RF data acquisition. Such synchronization may allow improved TF estimation.

The display of other TF parameters is possible. Most notably, in preliminary work, we were able to correlate the changes in TF phase with changes in viscosity. This may also provide image contrast. Unfortunately, because of sampling rate limitations, we have not collected data at high enough frequency to resolve phase lag. We plan to do this in the future.

In order to use the results presented in a needle insertion simulator suitable for brachytherapy treatment planning, a larger tissue volume will need to be imaged. We have been limited to the $50 \mathrm{~mm}$ depth in the present system due to hardware limitations of the US machine we are presently using. This limitation will dissappear as we upgrade the new Ultrasonix Sonix US machine.

\section{Conclusion}

We presented a system for the acquisition of patient specific visco-elasticity images of the prostate and peri-prostatic tissue using ultrasound vibro-elastography. 
The advantages of the approach include the acquisition of a measurement confidence measure through the coherence function, the ability to tune the frequencies at which measurements are taken and the ability to acquire repeatable, operator independent images. While only a few patients have been imaged so far, the results are encouraging. The measured frequency responses can be used to delineate the prostate and in needle insertion models and planners useful in brachytherapy treatment. Further research is required to determine the potential usefulness of such a system in prostate cancer diagnosis.

\section{References}

1. Zietman, A.L.: Localized prostate cancer: Brachytherapy. Curr Treat Options Oncol. 3(5) (2002) 429-36

2. Shao, F., Ling, K.V., Ng, W.S., Wu, R.Y.: Prostate boundary detection from ultrasonographic images. Journal of Ultrasound in Medicine 22 (2003) 605-623

3. Lagerburg, V., et al: Measurement of prostate rotation during insertion of needles for brachytherapy. Radiothera. Oncol. 77 (2005) 318-23

4. Alterovitz, R., et al.: Needle insertion and radioactive seed implantation in human tissues: Simulation and sensitivity analysis. In: Proc. IEEE Int. Conf. Rob. Aut. Volume 2. (2003) 1793-1799

5. Nag, S., et al: Intra-operative planning and evaluation of permanent prostate brachytherapy: Report of the American Brachytherapy Society. Int. J. Rad. Onc. Biol. Phys. 51(5) (2001) 1422-1430

6. Alterovitz, R., et al.: Sensorless planning for medical needle insertion procedures. In: Proc. IEEE/RSJ IROS Conf. Volume 4. (2003) 3337-3343

7. DiMaio, S.P., Salcudean, S.E.: Interactive simulation of needle insertion models. IEEE Trans. Biomedical Engineering 52(7) (2005) 1167-1179

8. Glozman, D., Shoham, M.: Flexible needle steering and optimal trajectory planning for percutaneous therapies. In: Proc. MICCAI. Volume 3217. (2004) 137-144

9. Goksel, O., et al: 3D needle-tissue interaction simulation for prostate brachytherapy. In: Proc. MICCAI. (2005) 827-834

10. Krouskop, T.A., Wheeler, T.M., Kallel, F., Garra, B.S., Hall, T.: Elastic moduli of breast and prostate tissues under compression. Ultrason. Imaging 20(4) (1998) 260-274

11. Klauser, A., et al.: Real-time elastography for prostate cancer detection. J. Urology 171(4 Suppl.) (2004) 477

12. Konig, K., et al.: Initial experiences with real-time elastography guided biopsies of the prostate. The Journal of Urology 174 (2005) 115-117

13. Turgay, E., Salcudean, S., Rohling, R.: Identifying mechanical properties of tissue by ultrasound. Ultrasound in Medicine and Biology 32(2) (2006) 221-235

14. Bendat, J.S., Piersol, A.G.: Random Data: Analysis and Measurement Procedures, 2nd Edition. Wiley Interscience. (1986)

15. Zahiri-Azar, R., Salcudean, S.E.: Time domain cross correlation with prior estimates. In: $3^{\text {rd }}$ Int. Conf. US. Meas. Imag. Tissue Elasticity, Cumbria, UK (2004)

16. Oppenheim, A., Schafer, R.: Digital Signal Processing. Englewood Cliffs, NJ, Prentice-Hall. (1975) 